\title{
Training Adults and Children with an Autism Spectrum Disorder to be Compliant with a Clinical Dental Assessment Using a TEACCH-Based Approach
}

\author{
Lorena M. Orellana $\cdot$ Sonia Martínez-Sanchis • \\ Francisco J. Silvestre
}

Published online: 4 September 2013

(C) Springer Science+Business Media New York 2013

\begin{abstract}
The specific neuropsychological and sensory profile found in persons with autism spectrum disorders complicate dental procedures and as a result of this, most are treated under general anesthesia or unnecessary sedation. The main goal of the present study was to evaluate the effectiveness of a short treatment and education of autistic and related communication-handicapped children-based intervention program (five sessions) to facilitate a 10-component oral assessment in children $(\mathrm{n}=38$, aged 4-9 years) and adults $(\mathrm{n}=34$, aged 19-41) with autism spectrum disorder (with or without associated intellectual disability). The assessment ranges from entering into the examination room to the evaluation of the dental occlusion. There were statistically significant differences in the number of components reached and in compliance before and after the training program.
\end{abstract}

Keywords Autism - Oral assessment - TEACCHbased training program $\cdot$ Compliance

L. M. Orellana · F. J. Silvestre

Department of Stomatology, University of Valencia,

Valencia, Spain

S. Martínez-Sanchis ( $₫)$

Department of Psychobiology, Faculty of Psychology,

University of Valencia, Avenida Blasco Ibáñez 21,

46010 Valencia, Spain

e-mail: sonia.mtnez-sanchis@uv.es

S. Martínez-Sanchis

Neurodevelopmental Disorders Research Unit,

University of Valencia, Valencia, Spain

F. J. Silvestre

Stomatology Unit, Dr. Peset University Hospital,

Valencia, Spain

\section{Introduction}

Autism spectrum disorder (ASD) is a lifelong neurodevelopmental disorder characterized by a neuropsychological profile which includes communication problems, proficiency in visual skills, enhanced attention to details (hyperselectivity), anomalous attention and sensory processing patterns, attachment to routines and, finally, difficulties in understanding temporal concepts (Mesibov et al. 2005). Many of these characteristics undergo the intense stress response exhibited by these persons in relation to several dental procedures, such as oral exploration, which require the opening of the oral cavity and this represents a considerable threat to patients in the autistic spectrum. Furthermore, they may even feel it is an aggressive intrusion and thus look for all possible mechanisms to protect this area. The probability of disruptive behavior is high, complicating, impairing or even preventing the dental intervention (Bäckman and Pilebro 1999a; Dávila and Jensen 1988; Friedlander et al. 2006; Green and Flanagan 2008; Klein and Nowak 1998; Luscre and Center 1996; Namal et al. 2007; Pilebro and Bäckman 2005; Shapira et al. 1989). Thus, the use of habitual dentistry material and techniques becomes extremely difficult and complex due to the existence of communication deficits, sensory modulation alterations and the attachment to invariable environments. This situation worsens if other comorbid conditions are present (ADHD, epilepsy or intellectual disability) (Friedlander et al. 2006; Green and Flanagan 2008; Loo et al. 2008). For all these reasons, these patients are submitted to general anesthesia or unnecessary high dose sedation, which may lead to different side effects such as nausea, vomits and obstruction of the superior respiratory conducts and other common anesthesia-associated risks (Boynes et al. 2010; Lalwani et al. 2007; Limeres-Posse 
et al. 2003; Mochizuki et al. 2007; Messieha 2009; Wang et al. 2012). Moreover, as a result of the above mentioned difficulties, these patients receive less dental care, which could result in more pathological dental conditions and a greater risk of loss of permanent teeth than other patients (Loo et al. 2008; Namal et al. 2007; Orellana et al. 2012).

The lack of understanding of medical explorations and/ or their consequences complicates the management of these patients by professional staff and parents. In fact, the majority of people with ASD present behavioral problems when receiving medical assistance. Furthermore, the level of daily stress is higher than in the general population because of communication deficits, poor understanding of social cues and the difficulty of generalization of what they have learned in a specific context. Compliance can be enhanced and both the stress response and subsequent disruptive behavior may be reduced by using the pedagogic strategies based on TEACCH (Treatment and Education of Autistic and related Communication-handicapped Children) principles. The TEACCH model is a professional training program and a clinical service for persons with ASD and their families throughout the whole life-span. It was developed at the University of North Carolina by Eric Schopler in 1972. The core treatment strategy is what is called Structured Teaching which is based on the idiosyncratic neuropsychological profile of people with ASD. Structured Teaching basically relies on temporal and spatial organization through visual information.

To date, in almost the majority of dental studies with this population, disruptive behaviors have been regulated using physical restraint, sedation and, even general anesthesia. Only a few works have employed protocols with different communicative strategies in children and toddlers to facilitate dental exploration and interventions (Bäckman and Pilebro 1999a, b; Cuvo et al. 2010; Luscre and Center 1996; Pilebro and Bäckman 2005; Yepes et al. 1998). In the past, to provide appropriate dental treatment, trials of clinical actions to be performed at home and in educational institutions have been suggested in order to anticipate and to familiarize patients with the tools and basic dental procedures (Kopel 1977; Swallow 1969). The use of modeling with in vivo models or via audio-visual methods has also been described to anticipate and improve the performance of clinical dental examination in these children (Luscre and Center 1996; Yepes et al. 1998). In Sweden, through the use of images and visual pedagogy based on the TEACCH model, they anticipate certain actions to facilitate oral hygiene (Pilebro and Bäckman 2005), dental examination and preventive dental treatment (Bäckman and Pilebro 1999a). Other methods described to facilitate dental care are aversive stimulus desensitization through successive approaches (Cuvo et al. 2010) and the technique of Tell-Show-Feel-Do, which informs the child what proceedings are to be carried out, and anticipate what he is going to feel (Jaber et al. 2011; Murshid 2005). All these studies have worked exclusively with a pediatric population.

This project aims at improving the quality of life of people with ASD and their families by granting access to non-invasive dental assessment in a familiar environment, using the TEACCH-based strategies they are accustomed to. This approach has the double purpose of reducing stress responses and enhancing compliance in these patients in dental settings. The main goal was to evaluate the effectiveness of a psychoeducational training program in facilitating a complete oral assessment.

\section{Methods}

\section{Participants}

A total of 114 persons with ASD ( 45 children and 69 adults) who were receiving clinical and educational support in specialized centers for people with autism and/or intellectual disability were initially recruited. The inclusion criteria to participate were: (a) having being diagnosed with autism, Asperger syndrome or Pervasive Developmental DisorderNot otherwise Specified; (b) children aged 4-10 years old and adults older than 18 (adolescents 11-17, were excluded to avoid hormonal factors which could influence results); (c) understanding visual or simple verbal instructions; (d) failure to undergo the 10 components of the oral assessment during the pre-intervention session; and (e) written informed parental consent. Thus, several subjects were discarded due to the following reasons: two children and 18 adults were able to complete the 10 steps of dental assessment; 5 children and 17 adults withdrew from at least one of the 5 sessions of the training program.

The final sample consisted of a total of 72 persons with ASD (38 children and 34 adults). The majority of the children ( 33 boys and 5 girls) who took part in the present study attended Mira'm Foundation in Valencia (Spain), which is an autism center and a specialized school for children with ASD, and the rest belonged to different associations or institutions (ASPAU, CEDIN, Psicotrade and Red Cross). The adults ( 29 men and 5 women, between 19 and 41 years old) came from the following centers in the Valencian Community and the Region of Murcia (Spain): $C A U$ ( $\mathrm{n}=13$, residential and day center for adults with ASD, Castellón); Angel Riviere ( $\mathrm{n}=5$, day center for adults with ASD, Valencia); Fuente de San Luis $(\mathrm{n}=4$, public day center for adults with intellectual disability, Valencia); Isabel de Villena $(\mathrm{n}=1$, public day center for adults with intellectual disability, Valencia); Juan de Garay 
( $\mathrm{n}=2$; public occupational center for adults with intellectual disability, Valencia); and Reina Sofía $(\mathrm{n}=9$, day center for adults with ASD, Molina de Segura, Murcia).

\section{Procedure}

Parents were offered a basic clinical oral assessment without cost for their sons or daughters with ASD, which would take place in each of the centers they attended. It was carried out by a dentist who is a specialist in attending patients with special needs. The study was approved by the ethics committee of clinical investigation at the University of Valencia, Spain.

Data about health, cognitive status and behavior was obtained by means of a questionnaire formed by 4 parts. Part A was directed to parents or tutors and gathered information in relation to associated pathologies, medication, and previous behavior with doctors; capacity to remain still for $5 \mathrm{~min}$; dental history and number of dentists visited; dental treatment and use of general anesthesia. Part B was for the educators, to obtain information on institutionalization, use of rewards, forms of communication, and teaching strategies. Part $\mathrm{C}$ was addressed to the psychologist in each center to collect information about cognitive development previously evaluated by standard tests used in this population. Lastly, part D was filled out by the dentist and corresponded to the dental case history of each subject, with sections on extra-oral and intraoral examination.

The study was planned according to a prospective quasiexperimental design and consisted of a baseline oral examination (pre-test), five training sessions and a final oral assessment (post-test). In both, pre- and post-tests, compliance and 10 consecutive steps necessary to carry out an appropriate oral assessment were evaluated. The oral evaluation ranged from entering the room to dental occlusion.

\section{Oral Assessment Steps}

\section{Entering the Room}

The subject enters the examination room when the dentist says "come in" alone or accompanied by the educator.

\section{Sitting Down in the Dental Chair}

The subject sits down in the chair and remains still for more than $10 \mathrm{~s}$ when the dentist says "sit down in the chair".

\section{Lying Back in the Chair}

The subject lies back without help and supports their head against the headrest with their legs stretched out or flexed when the dentist says "lie down in the chair".

\section{Tolerating Direct Light on the Face}

The subject is capable of tolerating the light focused on the chest when the dentist says "I am going to switch on the light". Later it is directed towards the face when the dentist says "I will put the light on your face", while the subject remains lying down.

\section{Opening the Mouth}

The subject is able to open the oral cavity to the maximum, maintaining it in this position when the dentist says "open your mouth". This is carried out twice.

\section{Tolerating Manipulation of the Mouth with Gloves}

The subject is capable of allowing the manipulation of the interior of his mouth with the fingers without closing it for intervals of at least $5 \mathrm{~s}$ when the dentist says "open your mouth; I will count your teeth with my fingers". Firstly, if necessary, the subject's fingers are counted with the dentist's fingers. This activity is performed twice.

\section{Examination with the Mouth Mirror}

The subject is able to tolerate the mouth mirror inside the oral cavity, keeping it opened for intervals of at least $5 \mathrm{~s}$ when the dentist says "open your mouth, I will count your teeth with the mirror". Firstly, if necessary, the subject's fingers are counted with the mirror. This activity is performed twice.

\section{Examination with the Probe}

The subject is capable of tolerating the probe inside the oral cavity, keeping it open for intervals of at least $5 \mathrm{~s}$ when the dentist says "open your mouth; I will count your teeth with the probe". Firstly, if necessary, the subject's fingers are counted with the probe. This activity is performed twice.

\section{Examination with Mirror and Probe}

The subject is able to tolerate the mirror and probe inside the oral cavity, keeping it open for intervals of at least $5 \mathrm{~s}$ when the dentist says "open your mouth, I will count your teeth with the mirror and the probe". Firstly, if necessary, the subject's fingers are counted with the mirror and probe. This activity is performed twice.

\section{Dental Occlusion}

The subject is capable of pressing together the upper and lower teeth for intervals of at least $5 \mathrm{~s}$, allowing 
manipulation with the fingers without opening the mouth when the dentist says "press your teeth together and show me them, I will count your teeth with my fingers". This activity is done twice.

\section{Compliance}

Frankl scale was used to measure compliance during the preand post-test (Frankl et al. 1962). This scale has been used to evaluate behavior of patients with ASD on their first visit to the dentist (Klein and Nowak 1999; Loo et al. 2008); during their dental examination (Harada and Nishino 2005; Murshid 2005); in their dental treatment (Harada and Nishino 2005; Loo et al. 2008; Lowe and Jedrychowsky 1987); during brushing their teeth (Harada and Nishino 2005); to evaluate predictors of compliance with dental treatment (Marshall et al. 2008) and, finally, to select patients for treatment under general anesthesia or sedation (Chaushu and Becker 2000; De Nova-García et al. 2007).

Data are divided into the following 4 categories: Definitively negative, negative, positive and definitively positive.

\section{Definitively Negative}

The patient rejects treatment, cries, fearful struggle, or any other evidence of extreme negativism.

\section{Negative}

Unwilling to accept treatment, non-compliant, some evidence of negative attitudes but not very pronounced.

\section{Positive}

The patient tolerates treatment cautiously with the desire to cooperate with the dentist, following the instructions and collaborating.

\section{Definitively Positive}

Good rapport with the dentist, interested in the dental procedure, smiles and enjoys the situation.

Evaluation in the Pre- and Post-test

Specific rooms were prepared in each center to be able to carry out the pre- and post-test. These were approximately $3 \mathrm{~m} \times 4 \mathrm{~m}$, with limited decoration and auditory stimulus to avoid distracting elements. A photograph of the dentist was placed in every daily timetable of the subjects, who were told that they would have an activity with this person, without specifying anything else. Later each subject was accompanied by their educator to the room where the test would take place. The dentist was waiting for them at the entry of the room with the pictographic sequence that indicated "today in the center the dentist is going to look at your teeth". In the rooms there was a folding dental chair, an articulated lamp for intraoral examination and a small table on which to exhibit the set of instruments. Material such as gloves and masks and a set of instruments like mirrors and dental probes were present. There was also a video camera with a tripod to film the entire activity for posterior assessment. For each of the 10 steps measured in the test, the dentist used simple verbal instructions and positive rewards afterwards. These steps were carried out sequentially and every component was rated as achieved or not.

\section{Behavioral Techniques Used in the Training Program}

Some of the behavioral strategies used in the training sessions are shown in Fig. 1.

\section{Successive Approaches}

Each of the subjects could get to know and interact (in a visual/olfactory/auditory/tactile form) with the set of dental instruments and with the dentist who carried out the examination (always dressed in the same way and with the same hairstyle).

\section{Tell-Show-Feel-Do $(T-S-F-D)$}

This is a common model of telling the patient what is going to happen, what he will feel or do, showing what would be done (Jaber et al. 2011; Murshid 2005). By means of this technique each of the 10 components of the oral examination was explained to every subject verbally in a simple form. Then visual, olfactory, auditory and/or tactile demonstrations were carefully made (using a model, a macro model or stuffed toys and a crocodile puppet for children) and, finally, the procedure was completed by carrying out the action with the subjects themselves; thus anticipating a certain clinical action.

\section{Visual Pedagogy}

By means of visual pedagogy, the subjects anticipated each of the steps in a visual form (photographs, pictographs, real objects) although it was also accompanied by short simple verbal sentences. To this end, a set of 20 photographs $(20 \mathrm{cms} \times 25 \mathrm{cms})$ were used, which showed sequentially each of the steps necessary to perform the dental examination. They included the room where the examination was to be carried out; the dental chair; the light and the set of dental instruments. Every photograph was accompanied by 

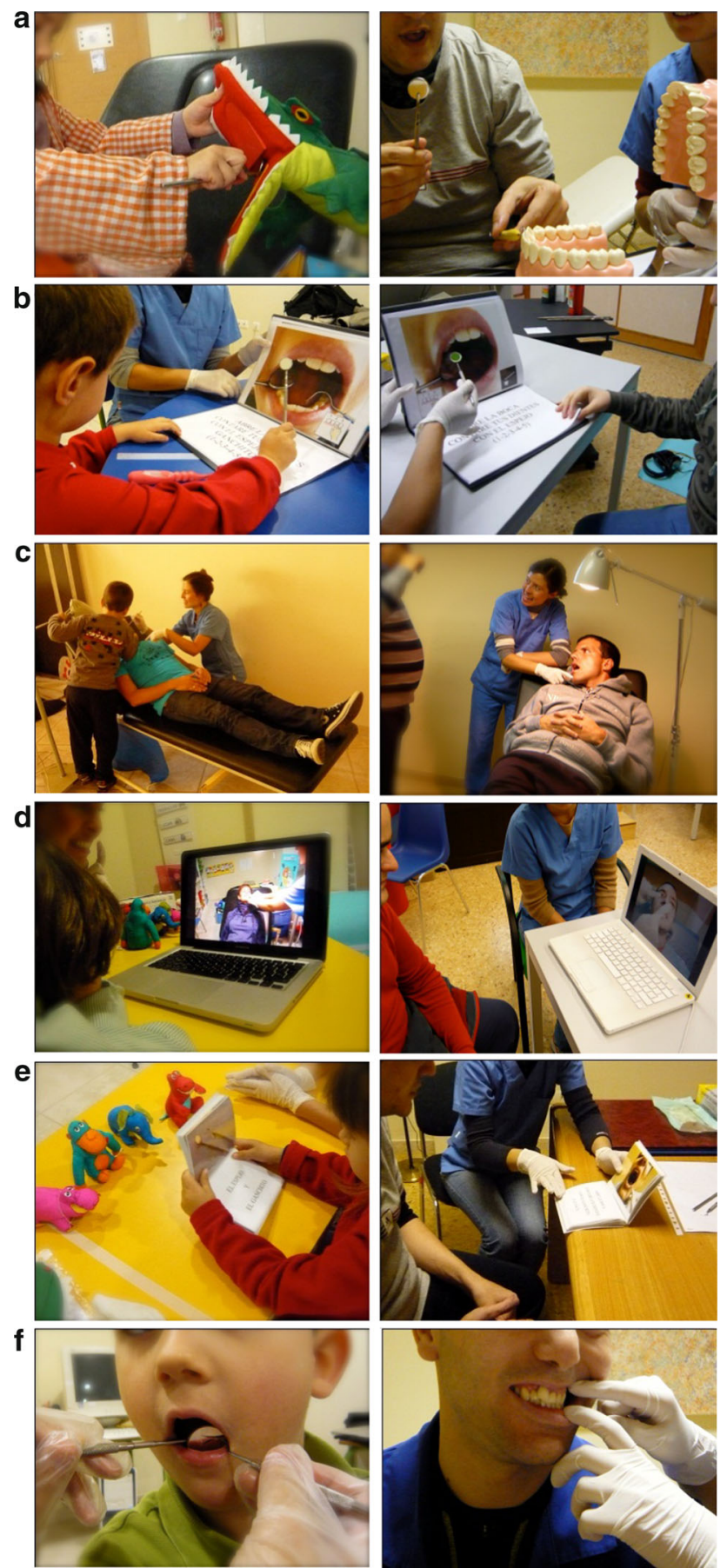

Fig. 1 Behavioral techniques used in training sessions; a successive approaches; b visual pedagogy; $\mathbf{c}$ in vivo modeling; $\mathbf{d}$ audio-visual modeling; e auto-modeling; $\mathbf{f}$ behavioral trials

a written instruction and pictographs related to the clinical action.

\section{In Vivo Modeling}

By means of in vivo modeling, the subjects were positioned in front of a model that showed the behavior that they were required to imitate. This took place in the room where the clinical examination was to be carried out. The educator or one of the most collaborating subjects was chosen to be submitted to this fictitious exploration so as to serve as a positive model to the rest of the group.

\section{Audio-Visual Modeling}

The same subject who performed the live modeling was filmed carrying out the 10 steps and later it was shown to the rest of the subjects. The video lasted 4 min approximately.

\section{Behavioral Trials}

Through behavioral trials, each of the 10 steps of the mouth examination was practiced sequentially, using elements or objects, such as the mouth mirror, which would be present in the real situation. This activity would facilitate the execution of these actions, since it would help them to foresee what is going to happen.

\section{Auto-Modeling}

In addition, photographs of subjects were taken during all the previous meetings while they were carrying out the targeted behavior. These images were edited or modified, eliminating the undesirable aspects. Later, subjects were instructed to observe and practice these actions.

\section{Training Sessions}

This training program was carried out in five 20-minute sessions, twice a week along three consecutive weeks.

For the training sessions, the materials used included the dentist's photograph laminated in plastic $(5 \times 5 \mathrm{~cm})$, and the set of photographs $(20 \mathrm{cms} \times 25 \mathrm{cms})$ which showed each of the 10-component dental assessments. There were two plastic macro models of the dental arcades and a dental brush and stuffed toys and a crocodile puppet for children. A set of real objects like mouth mirrors, probes of caries, gloves and masks was shown. There was also a computer and a camera to take photographs of each participant for the session of auto-modeling.

The structure of the sessions is shown in Table 1.

\section{Statistical Analysis}

Due to the nature of the design and data, which were not normally distributed, the paired sample Wilcoxon signed rank test was used to compare the results obtained between the pre- and post-test. The differences were considered statistically significant when $p<0.05$. The data analysis was performed with the program SPSS 18 for Windows ${ }^{\circledR}$. 
Table 1 Structure of the training sessions

\begin{tabular}{|c|c|}
\hline Session & Behavioral strategies \\
\hline 1 st & Successive approaches $+\mathrm{T}-\mathrm{S}-\mathrm{F}-\mathrm{D}^{\mathrm{a}}+$ visual pedagogy \\
\hline 2nd & $\begin{array}{l}\text { Successive approaches }+ \text { T-S-F-D }+ \text { visual } \\
\text { pedagogy }+ \text { audio-visual modeling }\end{array}$ \\
\hline 3 rd & $\begin{array}{l}\text { Successive approaches }+\mathrm{T}-\mathrm{S}-\mathrm{F}-\mathrm{D}+\text { visual } \\
\text { pedagogy }+ \text { in vivo modeling }\end{array}$ \\
\hline 4th & $\begin{array}{l}\text { Successive approaches }+\mathrm{T}-\mathrm{S}-\mathrm{F}-\mathrm{D}+\text { visual } \\
\text { pedagogy }+ \text { behavioral trials }\end{array}$ \\
\hline 5th & $\mathrm{T}-\mathrm{S}-\mathrm{F}-\mathrm{D}+$ auto-modeling + behavioral trials \\
\hline
\end{tabular}

a Tell-Show-Feel-Do

There were statistically significant differences regarding the number of components achieved in the dental assessment when comparing pre- and post-intervention in children $(\mathrm{z}=-5.254, p<0.001)$ and adults $(\mathrm{z}=-5.101$, $p<0.001$ ) (See Fig. 2).

Similar results were found when dividing the sample depending on the cognitive developmental level. A higher number of steps was achieved after the training program in high functioning children $(\mathrm{z}=-3.744, p<0.001)$ and in children and adults with mild $(\mathrm{z}=-2.677, p<0.01$; $\mathrm{z}=-3.643, p<0.001$, respectively) and severe intellectual disability $(\mathrm{z}=-2.701, \quad p<0.01 ; \mathrm{z}=-3.639$, $p<0.001$, respectively) (See Fig. 3).

With regards to behavior evaluated using the Frankl scale there were statistically significant differences between moments pre- and post-intervention in children $(\mathrm{z}=$ $-4.970, p<0.001)$ and adults $(\mathrm{z}=-5.245, p<0.001)$ (See Fig. 4). Similarly, higher scores were obtained after the training program in high functioning children $(\mathrm{z}=-3.682$, $p<0.001)$ and in children and adults with mild $(\mathrm{z}=$ $-2.460, p<0.05 ; \mathrm{z}=-3.758, p<0.001$, respectively) and severe intellectual disability $(\mathrm{z}=-2.414, p<0.05$; $\mathrm{z}=-3.758, p<0.001$, respectively) (See Fig. 5).

\section{Discussion}

The TEACCH-based training program used in the present study was effective in facilitating a full dental assessment by increasing compliance in children and adults with ASD, with and without intellectual disability. The dental
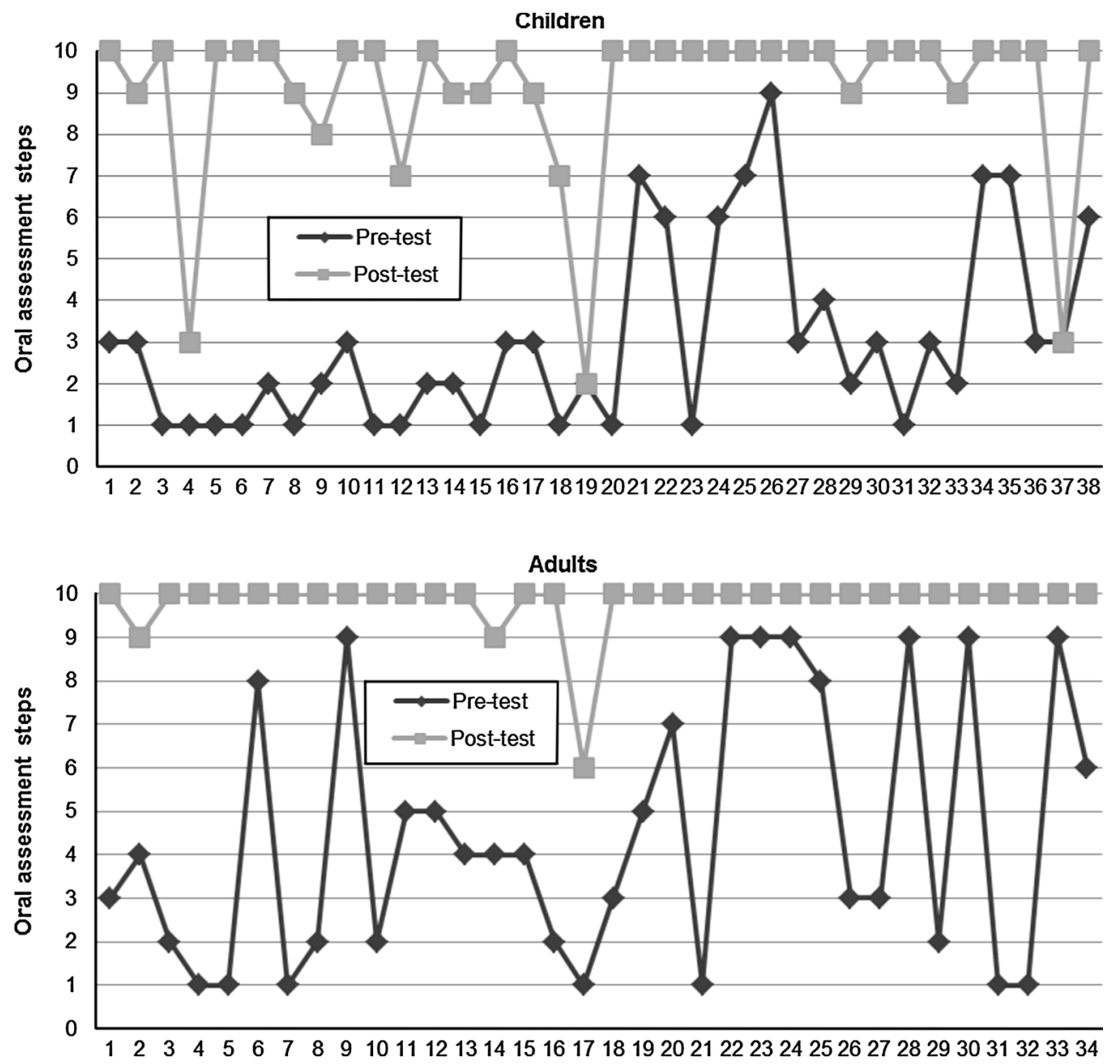

Fig. 2 Number of oral assessment steps achieved by children and adults in pre- and post-tests 
High-functioning children

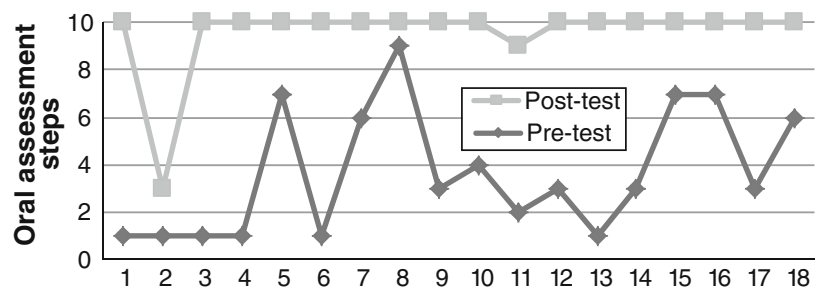

Children with mild intellectual disability

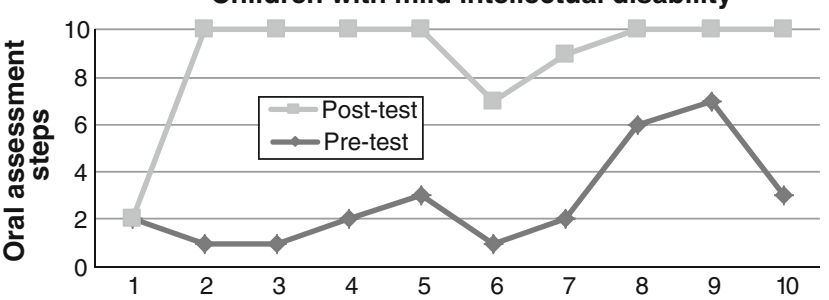

Children with severe intellectual disability

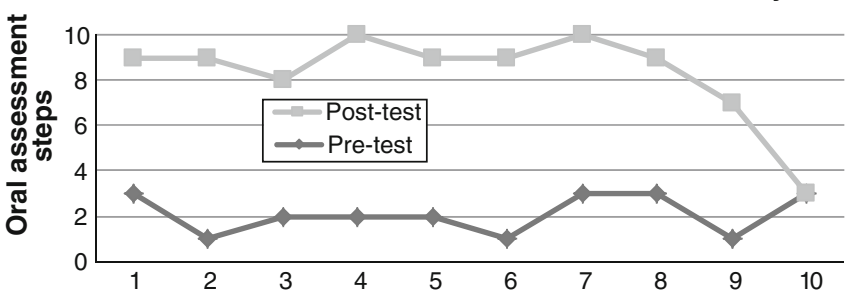

Adults with mild intellectual disability

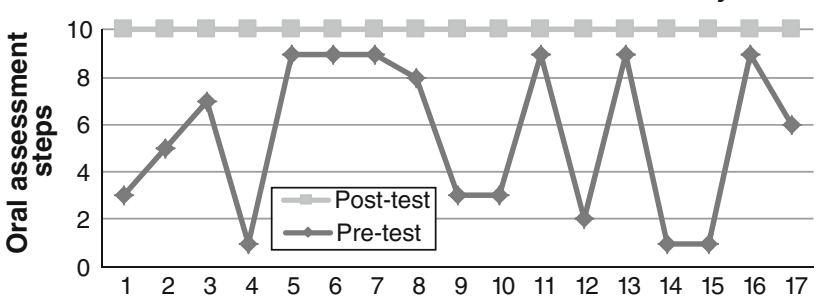

Adults with severe intellectual disability

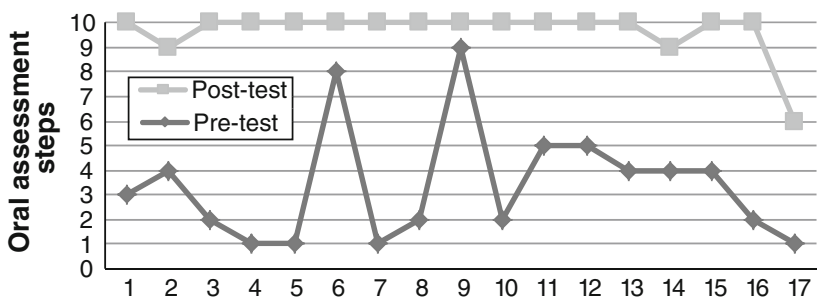

Fig. 3 Number of oral assessment steps achieved depending on cognitive status

examination composed of 10 steps ranged from entering into the examination room to the evaluation of the dental occlusion. Only after five training sessions (distributed along three consecutive weeks), the majority of the whole sample $(77.77 \%)$ was able to press the arcades together and $90.27 \%$ reached the penultimate step, which was the intra-oral examination with both the mirror and the probe. The development of this project demonstrates the efficacy of the psycho-educational techniques and strategies used in these patients with special needs. The improvement was not influenced by the level of cognitive development, indicating that both children with high functioning autism and children and adults with associated intellectual disabilities could benefit from this intervention.

Furthermore, keeping in mind the scarce literature on this subject, this study includes the largest number of subjects, a total of 72 patients of whom 38 were children and 34 adults. It is also worth noting that there are no previous studies that evaluate a psycho-educational approach in adults with ASD.

The assessment of pre- and post-test behavior was performed using Frankl's scale, which has been the most frequently used in the dental field to evaluate compliance in ASD patients. In the pre-test, $73.7 \%$ of children and $67.6 \%$ of adults showed a reluctant behavior (Frankl values 1 and 2); a similar percentage to that observed initially by Lowe and Jędrychowski (1987), Klein and Nowak (1999), Chaushu and Becker (2000), Marshall et al. (2008), and Subramaniam and Gupta (2011) with 70, 72, 66.6, 65, and $65 \%$, respectively. The rest of the studies described somewhat lower percentages of initial negative behavior in these patients (Loo et al. 2008; Harada and Nishino 2005). Only Murshid (2005) and De Nova-Garcia (2007) found higher rates of negative behavior (85 and $100 \%$, respectively). In the present study it was observed that the children had a slightly higher percentage of negative behavior in the pre-intervention group compared to adults.

In the post-test, $81.6 \%$ of our children and $100 \%$ of adults demonstrated positive behavior. There are no other studies comparing pre- and post-test behavior using Frankl's scale.

Best practice guidelines for persons with ASD promote actions whose goal is to improve the quality of life of both these persons and their families. Quality of life is founded on the subject's perception and it would depend on health, psychological status and the relationship with the environment. Emotional and physical well-being are some quality of life dimensions which would include both reduction of chronic stress response and access to preventive and specialized healthcare adapted to the special 
Fig. 4 Evaluation of behavior according to Frankl Scale in pre- and post-tests
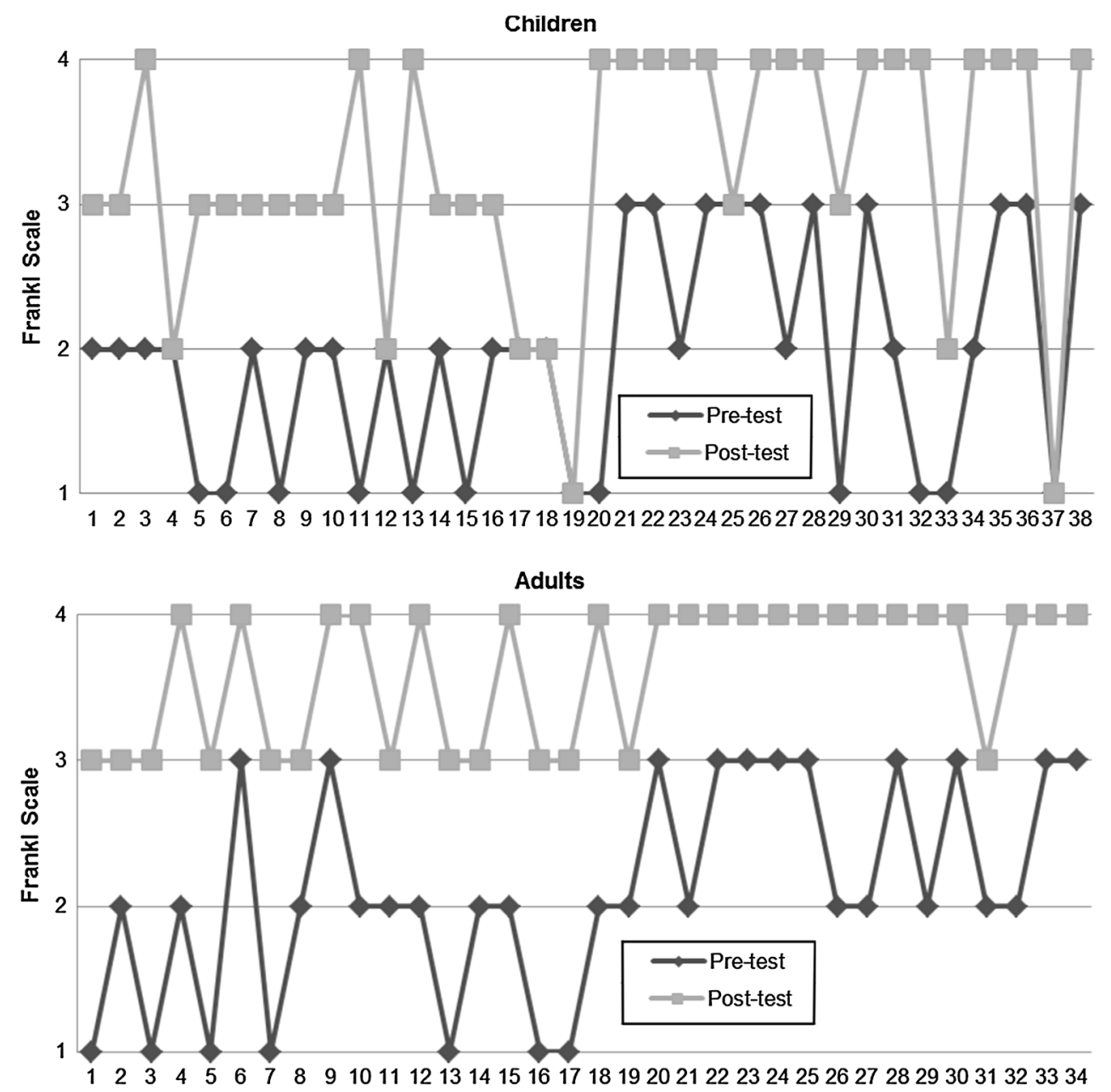

characteristics and needs of persons with ASD. The dental practice developed in the present study contributes to improve emotional and physical well-being in adults and children, facilitating acquisition and interchange of professional knowledge among dentists and specialized professional staff from the day care centers and proportioning an assistance training protocol which could be adapted to different healthcare examinations. As the first contacts with the dentist take place at the same day care centers they attend, this diminishes uncertainty and promotes adaptation to future dental interventions even in different environments. The final goal is to guarantee that persons with disabilities have equal opportunities in dental care settings. It implies teaching from the point of view of "culture of autism", e.g., taking into account the needs and way of processing information (including sensory profile) of persons with ASD. Thus, considering the effectiveness of this protocol, it could be taken into account as a guideline for the dental care of persons with ASD throughout their lifespan. Furthermore, it favors the emotional and physical trust of medical settings, which could be extended to other health care practices.
Within the limitations found in this study we could include the need of a follow up in order to evaluate compliance in a dental clinic. In a future study, it would be useful to incorporate the assessment of each training session and the effectiveness of each technique, taking into account the sensory profile of each participant so that every activity could be more individualized. In fact, the 12 children and 3 adults who failed to achieve the dental occlusion step had serious tactile hypersensitivity, so vinyl gloves instead of latex gloves were used with them.

We believe that to attend people with ASD, it is essential that health professionals be trained in the care of patients with special needs, it would be they who visited the day care centers in the first interventions, since a familiar environment favors collaboration. Subsequently, patients who go to the dental office, would only have to be given a preview of this new place, because they would be familiar with the professional and the actions to be carried out. It is therefore essential that during the first meetings, the dentist achieves a good rapport with their patient so as to inspire confidence and enable the objectives of the intervention to be reached. 

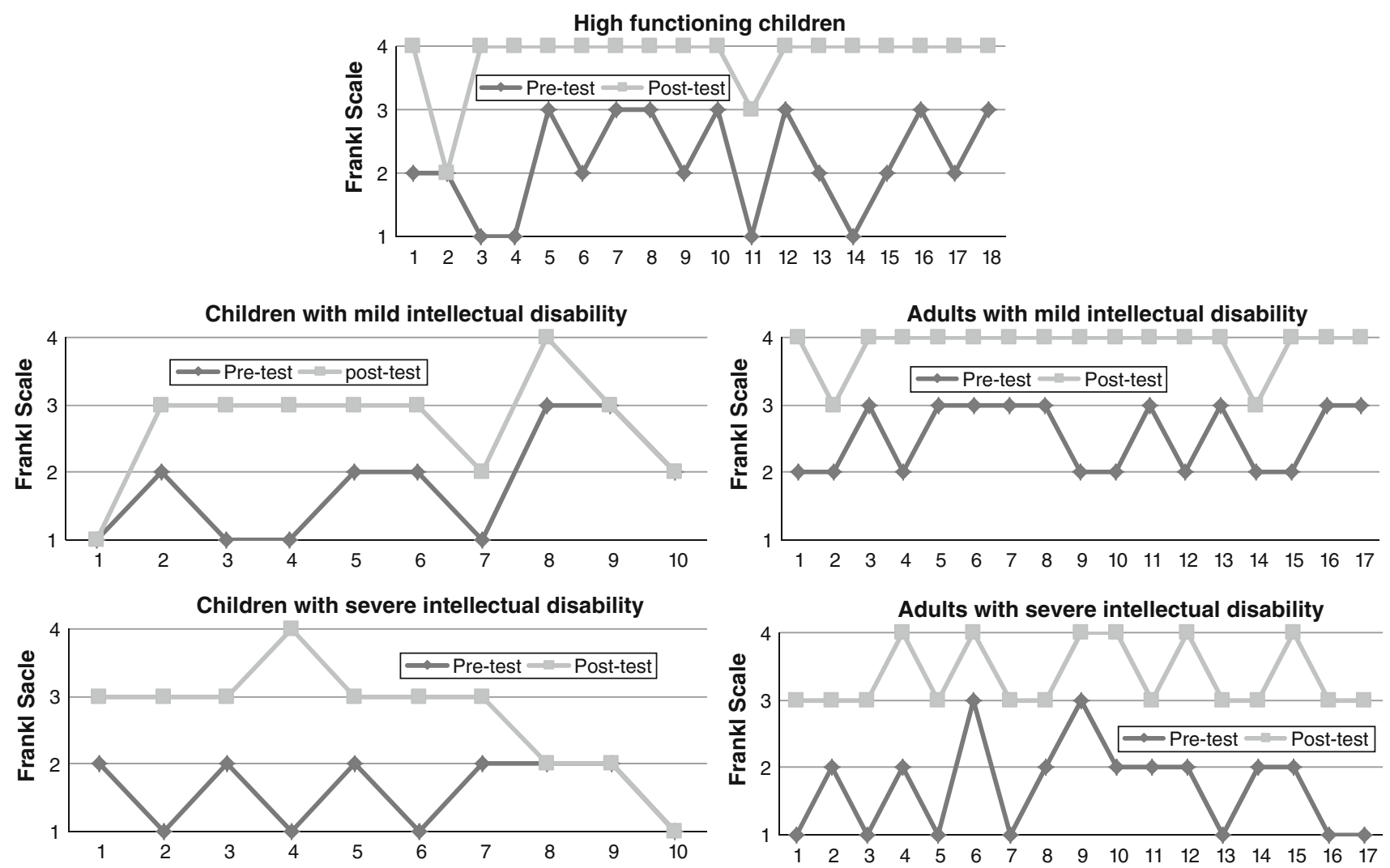

Fig. 5 Evaluation of behavior according to Frankl Scale depending on cognitive status

In summary, the results found in our study support the reliability of the TEACCH program as an evidence-based intervention (Mesibov and Shea 2010). The improvement in dental assessment after the training protocol constitutes a real-life outcome measure in a complex condition as is a dental care setting.

Acknowledgments The authors wish to thank all the children and adults who participated in the study and their families. They would like to express their gratitude for the collaboration to the following centers and associations: Mira'm Foundation; Project Autism Association (ASPAU); Autism Center (CAU, residential and day center for adults with ASD); Angel Riviere (day center for adults with ASD); Fuente de San Luis (public day center for adults with intellectual disability); Isabel de Villena (public day center for adults with intellectual disability); Juan de Garay (public occupational center for adults with intellectual disability); Reina Sofía (day center for adults with ASD); Red Cross; Psicotrade; Childhood Development Center (CEDIN).

\section{References}

Bäckman, B., \& Pilebro, C. (1999a). Visual pedagogy in dentistry for children with autism. Journal of Dentistry for Children, 66(5), 325-331.

Bäckman, B., \& Pilebro, C. (1999b). Augmentative communication in dental treatment of a nine-year-old boy with Asperger syndrome. Journal of Dentistry for Children, 66(6), 419-420.
Boynes, S. G., Moore, P. A., Lewis, C. L., Zovko, J., \& Close, J. M. (2010). Complications associated with anesthesia administration for dental treatment in a special needs clinic. Special Care in Dentistry, 30(1), 3-7. doi:10.1111/j.1754-4505.2009.00114.x.

Chaushu, S., \& Becker, A. (2000). Behaviour management needs for the orthodontic treatment of dental children with disabilities. European Journal of Orthodontics, 22(2), 143-149.

Cuvo, A. J., Godard, A., Huckfeldt, R., \& DeMattei, R. (2010). Training children with autism spectrum disorders to be compliant with the oral assessment. Research in Autism Spectrum Disorders, 4, 681-696. doi:10.1016/j.rasd.2010.01.007.

Dávila, J. M., \& Jensen, O. E. (1988). Behavioral and pharmacological dental management of a patient with autism. Special Care in Dentistry, 8(2), 58-60.

De Nova-García, M. J., Gallardo-López, N. E., Martín-San Juan, C., Mourelle-Martínez, M. R., Alonso-García, Y., \& CarracedoCabaleiro, E. (2007). Criteria for selecting children with special needs for dental treatment under general anaesthesia. Medicina Oral Patología Oral y Cirugía Bucal, 12(7), E496-E503.

Frankl, S. N., Shiere, F. R., \& Fogels, H. R. (1962). Should the parent remain with the child in the dental operatory? Journal of Dentistry for Children, 29, 150-163.

Friedlander, A. H., Yagiela, J. A., Paterno, V. I., \& Mahler, M. E. (2006). The neuropathology, medical management and dental implications of autism. Journal of the American Dental Association, 137(11), 1517-1527.

Green, D., \& Flanagan, D. (2008). Understanding the autistic dental patient. General Dentistry, 56(2), 167-171.

Harada, K., \& Nishino, M. (2005). Survey on management of oral health for autistic people. Dental Examination and tooth brushing. Dentistry in Japan, 41, 161-163. 
Jaber, M. A., Sayyab, M., \& Abu-Fanas, S. H. (2011). Oral health status and dental needs of autistic children and young adults. Journal of Investigative and Clinical Dentistry, 2(1), 57-62.

Klein, U., \& Nowak, A. J. (1998). Autistic disorder: A review for the pediatric dentist. Pediatric Dentistry, 20(5), 312-317.

Klein, U., \& Nowak, A. (1999). Characteristics of patients with autistic disorder (AD) presenting for dental treatment: A Surrey and chart review. Special Care in Dentistry, 19(5), 200-207.

Kopel, H. M. (1977). The autistic child in dental practice. ASDC Journal of Dentistry for Children, 44(4), 302-309.

Lalwani, K., Kitchin, J., \& Lax, P. (2007). Office-Based dental rehabilitation in children with special healthcare needs using a pediatric sedation service model. Journal of Oral and Maxillofacial Surgery, 65(3), 427-433.

Limeres-Posse, J., Vázquez-García, E., Medina-Henríquez, J., TomásCarmona, I., Fernández-Feijo, J., \& Diz-Dios, P. (2003). Preassessment of severely handicapped patients suitable of dental treatment under general anesthesia. Medicina Oral, 8(5), 353-360.

Loo, C. Y., Graham, R. M., \& Hughes, C. V. (2008). The caries experience and behavior of dental patients with autism spectrum disorder. Journal of the American Association, 139(11), $1518-1524$.

Lowe, O., \& Jedrychowski, J. R. (1987). A sedation technique for autistic patients who require dental treatment. Special Care in Dentistry, 7(6), 267-270.

Luscre, D. M., \& Center, D. B. (1996). Procedures for reducing dental fear in children with autism. Journal of Autism and Developmental Disorders, 26(5), 547-556.

Marshall, J., Sheller, B., Mancl, L., \& Williams, B. (2008). Parental attitudes regarding behavior guidance of dent al patients with autism. Pediatric Dentistry, 30(5), 400-407.

Mesibov, G. B., \& Shea, V. (2010). The TEACCH Program in the era of evidence-based practice. Journal of Autism and Developmental Disorders, 40(5), 570-579. doi:10.1007/s10803-009-0901-6.

Mesibov, G. B., Shea, V., \& Schopler, E. (2005). TEACCH approach to autism spectrum disorders. Raleigh, NC: Autism Society of North Carolina.

Messieha, Z. (2009). Risks of general anesthesia for the special needs dental patient. Special Care in Dentistry, 29(1), 21-25. doi:10.1111/j.1754-4505.2008.00058.x.
Mochizuki, K., Tsujino, K., Ohtawa, Y., Yakushiji, M., Nomura, K., Ichinohe, T., et al. (2007). Dental care for physically or mentally Challenged at public dental clinics. The Bulletin of Tokyo Dental College, 48(3), 135-142.

Murshid, E. Z. (2005). Oral health status, dental needs, habits and behavioral attitude towards dental treatment of a group of autistic children in Riyadh, Saudi Arabia. The Saudi Dental Journal, 17, 133-139.

Namal, N., Vehit, H. E., \& Koksal, S. (2007). Do autistic children have higher levels of caries? A cross-sectional study in Turkish children. Journal of the Indian Society of Pedodontics and Preventive Dentistry, 25(2), 97-102.

Orellana, L. M., Silvestre, F. J., Martínez-Sanchis, S., Martínez-Mihi, V., \& Bautista, D. (2012). Oral manifestations in a group of adults with autism spectrum disorder. Medicina oral, patología oral y cirugía bucal, 17(3), e415-e419.

Pilebro, C., \& Bäckman, B. (2005). Teaching oral hygiene to children with autism. International Journal of Paediatric Dentistry, 15(1), 1-9.

Shapira, J., Mann, J., Tamari, I., Mester, R., Knobler, H., Yoeli, Y., et al. (1989). Oral health status and dental needs of an autistic population of children and young adults. Special Care in Dentistry, 9(2), 38-41.

Subramaniam, P., \& Gupta, M. (2011). Oral health status of autistic children in India. The Journal of Clinical Pediatric Dentistry, 36(1), 43-47.

Swallow, J. N. (1969). The dental management of the autistic children. British Dental Journal, 126, 128-131.

Wang, Y. C., Lin, I. H., Huang, C. H., \& Fan, S. Z. (2012). Dental anesthesia for patients with special needs. Acta Anaesthesiologica Taiwanica, 50(3), 122-125.

Yepes, M., Ramírez, A., Restrepo, M. M., Sierra, M. C., Velásquez, M. I., Botero, M., et al. (1998). Aplicación de un método audiovisual para mejorar la comunicación con los niños autistas en el consultorio odontológico (Application of an audiovisual method to improve Communications with autistic patients in the dental setting). Revista CES Odontología, 11(2), 37-40. 